\title{
Theories: Tools versus Models ${ }^{1}$
}

\author{
Mauricio Suárez $^{2}$ \\ (Universidad Complutense de Madrid) and \\ Nancy Cartwright \\ (London School of Economics and University of California at San Diego)
}

Keywords: Theories, Models, Phenomena, Superconductivity, Structures, Instrumentalism

Abstract: In "The Toolbox of Science" (1995) together with Towfic Shomar we advocated a form of instrumentalism about scientific theories. We separately developed this view further in a number of subsequent works. Steven French, James Ladyman, Otavio Bueno and Newton Da Costa (FLBD) have since written at least eight papers and a book criticising our work. Here we defend ourselves. First we explain what we mean in denying that models derive from theory - and why their failure to do so should be lamented. Second we defend our use of the London model of superconductivity as an example. Third we point out both advantages and weaknesses of FLBD's techniques in comparison to traditional Anglophone versions of the semantic conception. Fourth we show that FLBD's version of the semantic conception has not been applied to our case study. We conclude by raising doubts about FLBD's overall project.

\section{Introduction.}

In 1994 the two of us together with Towfic Shomar wrote a brief position paper, "The Toolbox of Science", 3 advocating an instrumentalist view of theory: a good theory is

\footnotetext{
${ }^{1}$ Research towards this paper was supported by a grant from the New Del Amo Foundation, for which we are very grateful. We would also like to thank Gabriele Contessa, Sophia Efstathiou, Isabel Guerra and Albert Solé for help in its presentation.

${ }^{2}$ Corresponding author: msuarez@filos.ucm.es
} 
not primarily a collection of models (nor of claims) that cover the phenomena in its domain. When it comes to models of phenomena, theory is rather a tool for their construction.

The bulk of "The Toolbox" is an example from early attempts to understand superconductivity - a model by Fritz and Hans London. The example was further developed by one of us, who described its epistemological implications in greater detail (Suárez, 1995, 1997, 1999). Previous models had successfully treated the resistanceless flow characteristic of superconductivity but they were stymied by the discovery of the Meissner effect - the expulsion of electromagnetic flux when superconducting materials pass the critical temperature. The Londons deployed electromagnetic theory, taking the old models that treated superconductors as ferromagnets and shifting the analogy to think of superconductors as diamagnets. They borrowed equations from the old model - just enough to keep resistanceless flow - but left behind those from which the borrowed equations were originally derived, added features in accord with the new analogy, and at last accounted for both resistanceless flow and the Meissner effect in a single treatment. Here was an exciting piece of scientific advance, a powerful new model that could do what could not be done within the old models. Theory served to help construct the new model but the model, and the accompanying claims about superconductivity and its sources, were not already there, contained in the theory - perhaps only in some highly abstract form - waiting to be extracted.

Steven French and his collaborators, James Ladyman, Otávio Bueno and Newton Da Costa (hereafter FLBD) have since published at least eight papers and one book containing attacks on our claims and comments on this case study, which includes two extended discussions in this journal (French and Ladyman, 1997, 1998, 1999; Bueno, 1997; Da Costa and French, 2000, 2003; Ladyman, 1998, 2002, pp. 257-260; French, 1999; Bueno, French and Ladyman, 2002). For our defence we turn in part to FLBD themselves. What they say about the case is consistent with our account and in particular shares the features that matter for our argument. Moreover their own

\footnotetext{
${ }^{3}$ Cartwright, Shomar and Suárez (1995); this paper is hereafter referred to as "The Toolbox", or following French and Ladyman (1997) as "CSS".
} 
development of the semantic conception of theories - if it were to do what is advertised for it - shows one way that theories can function as tools.

What is at stake in our case study is a kind of piecemeal borrowing from an old model of superconductivity to the new London model; borrowing that takes some assumptions but leaves others behind. FLBD claim that our description of the early model - or of classical electromagnetic theory into which it fits - as a "tool" for the construction of the new model is mistaken, and that their alternative in terms of partial relations fits the case study much better. We shall argue that, contrary to what FLBD say, our description is correct; and that moreover the partial relations approach, if it could be successfully applied, should fit well into our description. But we shall also argue that the partial structures approach has not, after all, been applied to this case study and that there seems no straightforward way to apply it to do the jobs demanded.

In section 2 we explain and defend our main doctrine in "The Toolbox", namely that successful model building is generally non-theory driven. Section 3 reviews the specific case of the London model, and finds nothing in FLBD's account that contradicts our own. Section 4 describes FLBD's account of the relations among models in terms of partial structures, and points out that this is in principle not incompatible with our instrumentalism. However, in section 5 we show that the FLBD approach has not been applied to the relevant features of the case study, contrary to what they claim. In section 6 we provide a suitable structuralist account of our case study and show that it remains entirely silent on the relations that must obtain between theories and models. In other words the issues discussed by FLBD are irrelevant to the theoretical instrumentalism of "The Toolbox".

\section{The 'theory-driven view' of models vs. model autonomy.}

"The Toolbox of Science" aimed to support the claims behind Mary Morgan and Margaret Morrison's Models as Mediators book and the four-year research project 
that led to it: ${ }^{4}$ Models are autonomous from theory - that is, they play essential roles that are separate from any role they play in constituting theory. The title of the book comes from the slogan: 'Models mediate between theory and reality'. Morgan and Morrison themselves describe roles of this kind that have received little attention in the philosophical literature. Morrison studies a case in many ways similar to ours, the building by Ludwig Prandtl in 1904 of the boundary layer model for viscous fluids. This phenomenological model employs the formal tools of classical hydrodynamics and the Navier-Stokes equations, but "the important point is that the approximations used in the solutions come not from a direct simplification of the mathematics of the theory, but from the phenomenology of the fluid flow as represented in the model" (Morrison, 1999, p. 59). Morgan shows how the lessons to be learned from models need not depend directly on the structure of the model itself but rather on how it behaves under manipulations suggested by an accompanying narrative (Morgan, 1999, pp. 347-388).

"The Toolbox" aim was to prise apart theory from models at the place where they seem closest: when theory 'provides' models that are intended to represent phenomena and their origins reasonably accurately. Our concern then was with models that are intended to be reasonably accurate accounts of target phenomena and their sources, which we may call representative models. We argued against the idea that a good theory will in general already contain (albeit perhaps in some abstract form) the good representative models that it spawns. To that end we wanted to take as an illustration a hard case, a case where a new account was introduced with theory playing the central role. The London brothers' model of superconductivity is uncontroversially grounded in classical electromagnetic theory. Nevertheless, we argued, the theory did not contain the model in the relevant sense. Even here where theory and model are closest, it is best to think of theory as a tool.

The doctrine that we argued against is shared in both 'traditional' syntactic and semantic accounts of theory. On the syntactic version a theory is a set of claims and representative models are supposed to be models of the theory, models such that all the claims of the theory are satisfied and no additional assumptions are imported

\footnotetext{
4 "Models in Physics and Economics", Centre for the Philosophy of the Natural and Social Sciences (CPNSS) research project from 1993-1996.
} 
except ones legitimately grounded in a description of the phenomena to be represented. When it comes to semantic accounts, where theories are sets of models, Bas van Fraassen's version (Van Fraassen, 1989, esp. chapter 9) offers an exact analogue to this: A theory is a set of models and the representative models are to be found among these. In Ronald Giere's early version (Giere, 1988, esp. chapter 3) theory generally provides only ideal models, generally simple ideal models. To treat real, complex phenomena, more and more factors true of the real situation are added into the ideal model until a good enough representation of the phenomena is achieved. But, as in the conventional syntactic account, and much as in the accounts of deidealisation of the Poznan group (L. Nowak, 1980) additions to the model are to be legitimated by descriptions of the phenomena themselves. This is for a venerable reason: A good theory should not rely on ad hoc adjustments to get its accounts of the phenomena right.

On our proposal about the relation of the theory to the models we make to represent phenomena, the question of ad hoc adjustment does not directly arise. Theory does not often provide representations in the first place. Rather, it offers the tools for constructing representations. This means that an old hope for an easy legitimation of predictions is dashed: Evidence confirms theory, which in turn implies predictions; the predictions are warranted by the very evidence that confirms the theory. That route for warrant is given up when the predictions do not follow from the theory but require manoeuvres that are licensed neither by the theory nor by the facts of the situation. The version of the semantic account of theories defended by FLBD foregoes this desirable route for warranting predictions just as we do.

By now this case study is just one among many available in the literature that supports the same point. We should in particular like to note Mathias Frisch's study of the status of Lorentz's force law within classical electromagnetism (Frisch, 2000 and 2005), because it too involves classical electromagnetic theory; and it makes our central point even more dramatically, since in Frisch's example the resulting model is not only ad hoc with respect to the electromagnetic theory that spawns it but the target model is also incompatible with the theory. 
The London model is a pre-quantum model that draws upon classical electromagnetic theory, including Ohm's law. Ohm's law matters because the so-called acceleration equation follows from it and in earlier models it was the acceleration equation that allowed for the resistanceless flow of electricity that characterises superconductivity. The London model follows the discovery of a second phenomenon peculiar to superconductivity, the Meissner effect: when the critical temperature for a material to become superconducting is reached, all magnetic flux is expelled. That cannot happen given Ohm's law and the preceding acceleration equation. Yet we want to retain the consequences of the acceleration-equation model for resistanceless flow.

The London model manages to have its cake and eat it too. The acceleration equation implies a certain inhomogeneous equation (we shall call it $I$ ) for the magnetic field. London and London replace this inhomogeneous equation with the related homogeneous equation $(H)$. The resulting model is incompatible with Ohm's law and the acceleration equation, but it retains enough from these to admit resistanceless flow while at the same time allowing the expulsion of the magnetic field. There were, we noted, reasons for trying this particular manoeuvre. But there was nothing in the theory and the descriptions of different conducting materials that legitimates using Ohm's law for some materials while holding it in abeyance for those that turn out to be superconducting.

So the London model was ad hoc. This has two consequences. First, the ability of the vast evidence for electromagnetic theory to carry over to confirm the occurrence of the phenomena was undermined - though it may not have mattered in this case, since the two target phenomena (resistanceless flow and the Meissner effect) had independent experimental evidence. Second, the capacity of the success of the London model to confirm the theory used in its construction is eroded. It is nonetheless a triumph of theory to account for novel phenomena in this ad hoc way. But this is connected with the instrumental reliability of the theory rather than its truth (For more on instrumental reliability see Suárez, 1997, 1999, 2005). The theory must be used in novel ways to produce the result; the result is not already there buried with the theory. That supports our view that theories function as tools, not as sets of models already adequate to account for the startling phenomena that reveal their power. 
We called the view we criticised 'the theory-driven view' of models and we summarised our intentions thus:

\section{CSS General Claim:}

"We want to argue against the theory-driven view on the following ground: it is rarely the case that models of the phenomena are arrived at as deidealisations of theoretical models." (CSS, p. 5, ital. orig.)

About the London model in particular we concluded:

\section{CSS Specific Claim:}

“...The new equation [i.e. the inhomogeneous equation] is clearly not found by introducing correction-terms into the old equation...[nor is it] derived from basic electromagnetic equations with a set of different physical assumptions. In fact, from the point of view of the theory-driven view the restriction to the homogenous equation just looks ad hoc." (CSS, p. 11, ital. orig.)

We concluded "The Toolbox" paper thus: "What is needed is the recognition of the independence from theory, in aims and methods, of the scientific activity we have come to call phenomenological model building" (CSS, p. 11). This echoes the models-as-mediators slogan that models are generally 'autonomous' from theory.

At one place French and Ladyman report our conclusions in almost the same words (French and Ladyman, 1997, p. 374):

"Since the homogeneous equation cannot be obtained from the inhomogeneous one by introducing correction terms, nor by introducing different physical assumptions and deriving it from the basic equations of electromagnetism, [Cartwright, Shomar and Suárez] conclude that this example cannot be dealt with by the 'theory-driven' view [of models]." 
Yet, despite the correct report of our view above, FLBD often read our conclusion differently, as the claim that theory plays no role whatever in model building, and this is the basis for much of their criticism. We shall take up two specific instances. Consider first what French and Ladyman say of the shift from earlier models to the London model (French and Ladyman, 1997, p. 378):

"The claim that this shift occurred in a theory-independent way or without the help of theory can be understood in one or other of at least two distinct ways: first, that there are no correspondences at all between the relevant models, or secondly, and alternatively, that such correspondences exist but they do so only at a 'phenomenological' level."

Yet compare with what we actually said about how the change should occur on the view we oppose (CSS, p. 147):

"A new model had to be constructed to account for the Meissner effect. According to the theory-driven view of models there are two possible ways to do so. Either we introduce some correction factors well motivated from the point of view of physical theory...or we go through the derivation that took us from Maxwell's equations to the 'acceleration equation' model and revise some of the physical assumptions..."

So neither of the two ways they propose coincides with any of the two ways that we described. It seems that the 'theory-driven view' that we oppose has been misunderstood to be a much weaker claim than we defined it to be. A paper that explicitly set out to defend an instrumentalist role of theory in the building of models has been erroneously taken by FLBD to advocate a 'no-role whatever' view of theories.

“The Toolbox" was partly inspired by some of Ernan McMullin's writings (McMullin, 1985), in which he distinguishes between causal and construct 
idealization. ${ }^{5}$ Causal idealisations are simplifications carried out on the phenomena and their conditions of production directly; examples include shielding and other techniques to control experimentally potential interference factors. Construct idealizations are simplifications carried out on the theoretical description by ignoring some of the properties of the phenomenon described. The most widely used example is within classical mechanics, the single harmonic oscillator description of a pendulum, which ignores its friction.

McMullin argued that a realist attitude to theory need not require the model of the phenomenon to be a deductive consequence of the theory, but it must require the simplifications introduced in the description to be legitimated by theory or by an otherwise acceptable description of the phenomena. Otherwise, warrant will not travel from (the evidence for) the phenomenon to the theory. We agree with McMullin, for reasons that we already spelt out. The process whereby a theory gets applied to a phenomenon in a way that makes it a gradually more realistic description must involve a 'de-idealisation' by legitimated steps if the success of the model is to be taken as evidence for the truth of the theory and the reality of its entities.

Bueno, French and Ladyman (2002) also attribute to us a different sense of independence from theory than the one we defined. They suggest two ways in which models may be said to be 'autonomous', neither of which is on face value equivalent to, or entailed by, our notion of 'not theory-driven'. First, they suggest, a model may be autonomous in the absolute sense that no theory deductively entails it. Second, a model may be autonomous in the relative historical sense that the theory that inspires it has not yet been found (Bueno, French and Ladyman, 2002, p. 515):
“A model may appear 'autonomous' from high-level theory simply because it cannot be straightforwardly deduced from such theory ... Alternatively, a model may appear 'autonomous' in the sense that, at the time it was proposed, it was not clear how it might be obtained from high-level theory in a more or less direct way... In such cases the 'autonomy' can be regarded as relative and only temporary."

\footnotetext{
${ }^{5}$ For a detailed discussion of the connections between the Toolbox and McMullin's work see Suárez (1997, 1999).
} 
As for the first, we have been explicit that 'theory-driven' does not mean merely 'deducible from theory' (Suárez, 1997, p. 50; and 1999, p. 176). It rather has to do with how changes that cannot be deduced from pure theory are justified. As for the second, it might or might not agree with our notion of 'independent from theory', depending on what exactly is meant by 'obtained' and 'in a more or less direct way'. In that regard our claim, like McMullin's, is more precise: The model is independent from theory if it was not built as a de-idealization of high-level theory by improvements legitimated by independently acceptable descriptions of the phenomena. This notion of 'independent' is of course historical, and hence the autonomy that grounds it may well be only temporary. ${ }^{6}$

In some places it looks as if FLBD's objection to our paper is not that our position is mistaken but rather that is now almost universally accepted. For instance in a review of thirty years of work on models, Da Costa and French remark (Da Costa and French, 2000, p. 121): "Surely no one in their right minds would argue that all model development in the sciences proceeds deductively". If they mean 'purely deductively' we agree. But if they mean the model should be consistent with theory and all additions and deletions and corrections should be independently motivated, then we do not think this is such a daft view after all. Perhaps no-one in their right minds would argue that all model development is this way, but the theory-driven view argued that it should be in the cases where theory can be taken either to explain or legitimate a model, and for good reason, as we have said: If the features of the model cannot be derived from the theory using legitimated descriptions of the target system then the warrant that a successful theory earns by having vast evidence in its favour cannot be passed on to the model. This is a high price to pay, so the theory-driven view should not be given up too readily.

Nor is it clear that FLBD are themselves so ready to jettison the theory-driven view. According to Bueno, French and Ladyman (2002, p. 515) when it is "not clear how ... [a model] might be obtained from high-level theory in a more or less direct way" this fact is frequently lamented by the model-builder and is very often only temporary.

\footnotetext{
${ }^{6}$ Cf. The discussions in CSS, p. 142 and Suárez, (1999a) p. 194.
} 
And their overall tone suggests that it is altogether a good thing that it is temporary. On the other hand FLBD endorse a theory of pragmatic truth that demands only that the model of the phenomena fit a theory in order for the claims of the model to be pragmatically true 'according to' the theory (Mikeberg, Da Costa and Chuaqui, 1986, p. 204; and Da Costa and French, 1990, pp. 256-257). Perhaps we should conclude from this that they, like us, have a mixed attitude to the strict demands of the theorydriven view: It is powerfully useful for theories and models to relate as the view demands and hence satisfying the demands of the theory driven view is ceteris paribus a desirable aim. But it cannot be taken as the norm for good scientific practice.

\section{The London model}

Far from contradicting our theses, much of what FBLD have written in the last 10 years in fact supports them. Let us not turn to French and Ladyman's (1997) original reply to our paper. In their discussion of the London model they offer four different kinds of considerations in opposition to "The Toolbox". In our view these all support rather than undermine our reservations about the 'theory-driven view'.

First is the rich history they tell from the Londons' papers and the studies by Kostas Gavroglu (1995) and P.F.Dahl (1989). This history stresses the importance of background knowledge, hunches and points of view that affect the final form of the model, and what Fritz London thought about it. FLBD explicitly admit the importance of these other theoretical elements in construction of representative models. They label them 'heuristics' and argue that "Coupled with an appreciation of the heuristic moves involved in scientific work, we believe that it [the partial structures approach] provides a powerful representational tool which offers the possibility of a unitary approach to the nature and role of models in science" (Bueno, French and Ladyman, 2002, pp. 516-517).

Most of the original documents referred to by French and Ladyman are the same as those that we used in the four-year-long study that led to our account and their reading of the history is the same as ours and as Gavroglu's and Dahl's on the points at issue. 
Most centrally for our criticism of the theory-driven view, French and Ladyman highlight the fact, which we describe in our work and which the Londons explicitly remark, that the London model substitutes for the old analogy of superconductors with ferromagnets a new one-superconductors are like diamagnets. This is important for the kinds of reasons Mary Morgan often points out. ${ }^{7}$ The analogy helps us to understand how the Londons work with their model and what they do and do not do with it: which assumptions they add and which not, what approximations they make and what fragments of electromagnetic theory they deploy. A formal reconstruction of the model on its own cannot help us to understand that.

French and Ladyman also take up a second point of Gavroglu's, that in 1950 Fritz London rejected the description of his model as 'phenomenological' on the grounds that, in London's own words, "It actually goes beyond what is given by measurements on matter in bulk. We replace the observation $\mathbf{B}=0$ by a quite definite hypothesis concerning the fine structure of the currents and the fields." (French and Ladyman, p. 384). They also note as well that by that time Fritz London was stressing a microphysical reduction programme suggested by the Londons' diamagnetic analogy that was "a valuable heuristic" (French and Ladyman, p. 388) for the quantum account of superconductivity in terms of long-range order and Cooper pairs.

They then draw two conclusions:

(i) "The distinction made by Fritz London, as early as 1935, was not between 'phenomenological' models and 'fundamental' theories in Cartwright's sense, but between 'macroscopic descriptions' or 'interpretations', involving the standard electromagnetic field quantities, and 'microscopic' ones." (French and Ladyman, p. 388).

(ii) "We conclude that even then [in 1935 and 1937] he [Fritz London] was concerned to situate this work outside of the phenomenological framework." (French and Ladyman, p. 388).

\footnotetext{
${ }^{7}$ Particularly saliently in Morgan, Models as Modelling, manuscript.
} 
Neither of these conclusions opposes our claims. In "The Toolbox" we use the word phenomenological only twice, both times in our penultimate paragraph: “...[w]e feel we have stumbled upon an example of phenomenological model building about which the theory-driven view has little to say. What is needed is the recognition of the independence from theory, in methods and aims, of the scientific activity we have come to call phenomenological model building." (CSS, p. 148). In keeping with the thesis of our paper, phenomenological model building here means the building of models that while providing accurate representations, do not follow from theory either by de-idealisation or by introducing otherwise acceptable descriptions of the facts. ${ }^{8}$ The London model is certainly phenomenological in this sense, as we claimed. This does not mean that it cannot also be phenomenological in some other sense. And indeed it seems that there are three distinct and independent senses of 'phenomenological' at issue here: i) a model that represents phenomena, ii) a model whose construction has not been theory driven, and iii) a model that derives purely from measurement. We focused mainly on ii) while FLBD often follow Fritz London's in focusing on iii). However iii) is not opposed to the theory-driven view, so it can not be part of our own instrumentalist view.

The second kind of consideration French and Ladyman offer to support their criticisms of our paper is a detailed review of the derivation in the London model. They say: "We have gone through these results in pitiless detail in order to indicate the extent of the structure which is retained in this example of theory change. We see that the broad theoretical context is still provided by Maxwell's equations..." (French and Ladyman, p. 381). Of course, we agree that the broad theoretical context is provided by Maxwell's equations — this was our starting point.

Moreover, describing the derivation in more detail as Suárez $(1997,1999)$ and French and Ladyman (1997) do, bears out our claim. The London model could be counted as a development of earlier models of superconductivity in that it makes both deletions (those assumptions involved in taking superconductors to be like ferromagnets) and

\footnotetext{
${ }^{8}$ This is also, incidentally, the sense in which Cartwright used the term in her (1983) and generally thereafter. The sense that FLBD employ is instead much closer to Heinz Post's (1971). In his essay review of Gavroglu's biography of Fritz London, French (1999) also criticised Gavroglu , and us as well in passing, for describing the London model as 'phenomenological' - this suggests that Gavroglu uses the term in a sense much closer to ours.
} 
additions (those required to take them to be like diamagnets). But these 'corrections' are ad hoc. For we do not find anywhere in the details of the London model a new description of the physical structure of superconducting materials that licenses jettisoning Ohm's law and introducing the inhomogeneous equation. That had to await the quantum treatment by Bardeen, Cooper and Schrieffer, the so-called BCS theory (Bardeen et al., 1957), which pictures a superconductor as a Bravais lattice in a sea of free electrons.

In the quantum model the differences in energy between initial and final states of the electrons interacting with the lattice are small enough that the overall potential between the electrons in the lattice and the free electrons is attractive, rather than repulsive as when the Coulomb potential alone is at work. This allows for the formation of Cooper pairs and ultimately for an account of both resistanceless flow and the Meissner effect. Thus the quantum model, unlike that of the Londons, grounds its equations in a description of the materials in which they are supposed to apply, a description of them as materials whose microconstitution is that of a Bravais lattice in a sea of free electrons.

(Of course, like any model BCS has flaws of its own. With respect to the issues at stake here, a really good model from the theory-driven point of view would include further measurable features that follow from the postulated lattice structure beyond the two features of superconductivity to be accounted for. This would provide independent means for identifying the structure postulated to explain the two superconducting phenomena and thus provide further assurances that the explanation is not ad hoc.)

The third kind of consideration French and Ladyman raise against "The Toolbox" is a pair of related theses on theory change drawn from the work of Heinz Post (1971). Post maintains first that experience and experiment limits the choice of models when theories change but does not determine it; and second that even in radical theory change much of the lower-level structure remains intact. We agree entirely. If one thinks of the construction of the London model as a change in theory rather than new model building, then neither Post's views, nor ours, would see the changes as theorydriven in our sense. The discussion is somehow beyond the point since ours is not a 
thesis about radical structural discontinuity but about the use of theories as instruments in building models.

\section{How theories and models relate, according to FLBD.}

The last set of considerations French and Ladyman produce against our claims are based on their own positive version of the semantic conception of theories. They maintain that, given the tools for formal representation they develop, one need not see theories as mere tools for models. Rather, with their methods it is possible to express the relation between the theory and a model, or between two models, formally and always in the same way. We agree that the new relations they allow in terms of partial structures add some flexibility to the usual Anglophone versions of the semantic conception. ${ }^{9}$ But we disagree that partial structures do what they are advertised to do. In particular, contrary to FLBD's repeated claim, they have not been applied to the superconductivity example. We turn to the general issue in this section, and to the particular application to superconductivity in the next section.

FLBD share the view of the bulk of semantic theorists that models are to be reconstructed as ordered n-tuples of sets: a set of objects; a set of properties, quantities and relations over these objects; and a set of functions on the quantities. ${ }^{10}$ Where they part company from other semantic theorists is with respect to how models relate to each other and to the world. (We should note that when FLBD talk about the relation of models to the world they always mean the relation of one model to another, possibly more complete or more accurate, model of the world.)

Like many semantic theorists, FLBD demand isomorphisms between two models. But for them the isomorphism need not be complete. In their first seven papers, they instead urge the notion of a partial isomorphism, where the partialness derives from

\footnotetext{
${ }^{9}$ Although they do not seem to add that much to the highly nuanced theory-nets of the German structuralist school. See e.g. Balzer, Moulines, Stegmüller (1987), Balzer and Moulines (1996), Gähde (2002).

${ }^{10}$ They usually drop the functions when they offer formal definitions. We shall follow them in this practice since functions can normally be defined in terms of further relations.
} 
the fact that the relation is between partial structures only, where a partial structure is one that employs partial relations. ${ }^{11}$ A partial structure $\left\langle\mathrm{D} ;\left\{\ldots ; \mathrm{R}^{\mathrm{n}}{ }_{\mathrm{i} 1}, \mathrm{R}^{\mathrm{n}}{ }_{\mathrm{i} 2}, \mathrm{R}^{\mathrm{n}}{ }_{\mathrm{i} 3} ; \ldots\right\}\right\rangle$ defines for each n-placed relation $R_{i}{ }_{i}$ a set of n-tuples over $D\left(R^{n}{ }_{i 1}\right)$ that satisfy $R_{i}{ }_{i}$, a set of n-tuples $\left(R^{n}{ }_{i 2}\right)$ that do not satisfy $R_{i}^{n}$, and a set of $n$-tuples $\left(R_{i 3}{ }^{n}\right)$ for which it is not defined whether they satisfy $\mathrm{R}_{\mathrm{i}}^{\mathrm{n}}$ or not. They define partial isomorphism only for a 2-placed relation but the generalization is clear. Given two partial structures $\mathrm{A}=$ $\left\langle\mathrm{D} ;\left\{\ldots ; \mathrm{R}_{\mathrm{i} 1}, \mathrm{R}_{\mathrm{i} 2}, \mathrm{R}_{\mathrm{i} 3} ; \ldots\right\}\right\rangle$ and $\mathrm{B}=\left\langle\mathrm{E} ;\left\{\ldots ; \mathrm{R}^{\prime}{ }_{\mathrm{i} 1}, \mathrm{R}^{\prime}{ }_{\mathrm{i} 2}, \mathrm{R}^{\prime}{ }_{\mathrm{i} 3} ; \ldots\right\}\right\rangle$ then "the function $\mathrm{f}: \mathrm{D}$ $\rightarrow \mathrm{E}$ is a partial isomorphism if $\mathrm{i}$ ) $\mathrm{f}$ is bijective, and ii) for every $\mathrm{x}$ and $\mathrm{y} \in \mathrm{D}, \mathrm{R}_{\mathrm{i} 1}(\mathrm{x}, \mathrm{y})$ if and only if $\mathrm{R}^{\prime}{ }_{\mathrm{i} 1}(\mathrm{f}(\mathrm{x}), \mathrm{f}(\mathrm{y}))$ and $\mathrm{R}_{\mathrm{i} 2}(\mathrm{x}, \mathrm{y})$ if and only if $\mathrm{R}^{\prime}{ }_{\mathrm{i} 2}(\mathrm{f}(\mathrm{x}), \mathrm{f}(\mathrm{y}))$ ). (Bueno, 1997, p. 596; French and Ladyman, 1999, p. 108).

FLBD then go on to make strong claims in favour of their notions of partial relation and partial isomorphism (and the recent addition of partial homomorphism that we discuss at the end of section 4). First, the use of partial relations will allow all (or the bulk of) models in science to be represented as set-theoretical structures, from the abstract models that are supposed to constitute theory on the semantic view, to iconic and material models such as mice and billiard balls. For instance, French and Ladyman (1999) write: "Our central claim is that to present iconic models, material models, and so on ... is to present certain (partial) set-theoretical structures ... the issue is how ... [models] should be represented so as to best capture relevant aspects of 'practice'." (French and Ladyman, 1997, p. 107). ${ }^{12}$

We do not disagree with the claim that for some purposes models can be represented as set-theoretical structures, especially since FLBD acknowledge that set-theoretical structure is not everything and heuristics (of different sorts for different tasks) play an important role. But we find the claim that these structures can capture much about practice implausible. What a set-theoretical model can do is to represent a set of claims: It presents a set of objects and exhibits relations that are supposed to hold for those objects. So all of scientific practice that does not consist in the making of claims gets left out. In the London case, however, what matters most are the claims of the

\footnotetext{
${ }^{11}$ First introduced by Bueno (1997).

${ }^{12}$ See also, for example, Da Costa and French (2000), p.120 who maintain that even supposing "it is true that there exist models which were developed in a manner that was independent of theory, still they can be represented in terms of [set-theoretical] structures".
} 
London model, so we will not quarrel on these grounds with the attempt to represent the theory as a set-theoretic structure.

This brings us to the second of FLBD's claims. With the aid of the concepts of partial relations, partial isomorphisms, partial truth and structure hierarchy, ${ }^{13}$ FLBD claim to be able to "provide a unitary account of models in science capable of encompassing their diverse forms and uses" (French and Ladyman, 1999, p. 104). The project depends on casting all the various uses of models as cases of correspondences between structures. For instance, the use of a model to provide accurate predictions in an experiment might get cast in terms of a correspondence between a model of the experiment, and a data model, as represented by means of partial structures. Again, we maintain that this inevitably leaves out a great deal of the very scientific practice that we are interested in, and that FLBD say that they too want to describe; we shall illustrate this when we attempt to apply their methods to the London model in the next section.

The job at issue is how to represent the piecemeal borrowing that often takes place in the construction of a new model or new theory, in line with Heinz Post's ideas of theory change and our account of the London model. ${ }^{14}$ This much is central to evaluating FLBD's claim to offer an account of all model use in science: only some objects and some features from the first model appear in the second, and the second will have new objects and new features different from, and generally incompatible with, those in the first. This is how [a model of] a plastic and wood construction can model a DNA chain in the same sense in which the ideal pendulum models an actual pendulum. Not all objects in the plastic and wood construction [for example, the

\footnotetext{
${ }^{13}$ These concepts are defined in various places. They can all be found together in French and Ladyman (1999) and in Bueno, French and Ladyman (2002), except for pragmatic truth, which is formally defined in Mikenberg, Da Costa and Chuaqui (1986) and characterised less formally in, interalia, Da Costa and French (1990).

${ }^{14}$ French, Ladyman and Bueno (2002) are explicit in claiming that the notion of partial isomorphisms captures the idea that two models "may share parts of their structure" (p. 499). So too are Da Costa and French (2000), who explain that in cases of approximation "some of the properties and relations of the system being modelled might be ignored" (p. 260) and also "vice-versa" (p. 261). French and Ladyman (1999) also talk of "ignoring" relations from one model in considering its relation to the other as well as explaining that for two related models there need be only a "correspondence between certain elements of the family $R_{i}$ [from the one model] and certain elements $R_{i}$ ' [from the second model]". French and Ladyman (1997) write in exactly the same way about the London model, taking note of the extent of the structure which is retained from the earlier models that treated superconductors as ferromagnets.
} 
screws] map onto objects in the DNA chain, nor do all the properties [for example, the colours]. Similarly not all the functional relations true of the ideal pendulum need be true of the actual one.

It should also provide a way to represent how theories behave from a classical instrumentalist view. For a classical instrumentalist the models that constitute theory represent a target situation if a part of the model matches part of the situation - the part that is supposed to describe the observable features of observable objects. It does not matter whether the theoretical account of the sources of the observable phenomena is wildly off base or not. In the same way, the advertisements for FLBD's methods suggest they should be able to capture the use of 'as if' models in economics notoriously defended by Milton Friedman. ${ }^{15}$ These are models that make highly unrealistic assumptions but generate selectively good predictions about specific matters of interest, though they may well be dramatically inaccurate in their predictions about other readily observable phenomena that the model does not aim to treat.

It seems then that we all agree then that piecemeal borrowing is a central practice in scientific modelling. The problem for FLBD is just that, as far as we can see, this piecemeal borrowing cannot after all be represented with partial isomorphisms. We turn now to the London example to illustrate this.

\section{Troubles for FLBD methods.}

We now focus on French and Ladyman's (1997) claim to be able to offer an alternative account of the London model that both shows our thesis that theories work like tools to be false, and that is also better suited to our case study. We shall argue that FLBD's methods do not seem to allow us to represent the relations between the London theory and the previous acceleration-equation theory, contrary to their claim, nor are these methods able to capture the relation between the general background

\footnotetext{
${ }^{15}$ In "The Methodology of Positive Economics" in Friedman (1953), pp. 3 - 43.
} 
theory - Maxwell's electromagnetic theory - and the more specialized London theory of superconductivity.

The key difference between the two models, as described both by us and by French and Ladyman, is that the older theorists assume superconductors to be ferromagnets and hence required the acceleration equation and, correlatively, the inhomogeneous equation, to be satisfied, while the Londons, in the aftermath of Meissner's discovery, assume instead that superconductors are 'like' diamagnets and thus require the homogeneous equation to be satisfied. To explain the problem we see with the FLBD methods, we shall present our own very highly abbreviated and caricatured structural reconstruction of the two accounts; first with a straightforward Suppes-style representation, then using the FLBD notion of partial relations and partial morphisms, to try - unsuccessfully, we think - to circumvent the problems that arise with the Suppes-style representation.

Let us suppose that the systems under study in both the earlier models and the London models are all and only superconductors. Since classical electromagnetism is the relevant background theory, we shall consider two quantities, $\mathbf{E}(\mathrm{x}, \mathrm{t})$ and $\mathbf{B}(\mathrm{x}, \mathrm{t})$, the electric and magnetic fields across the superconductor, which are represented in the model in the usual way as functions from space-time points to field values. ${ }^{16}$ So our representation of the theory associated with the earlier acceleration equation can be given thus:

$\mathrm{AET}=\langle\mathrm{S} ;\{\mathbf{E}(\mathrm{x}, \mathrm{t}), \mathbf{B}(\mathrm{x}, \mathrm{t})\}\rangle$ such that $\mathrm{P}$,

where $\mathrm{P}$ is the set of principles governing the $\mathbf{E}$ and $\mathbf{B}$ values of the objects in $\mathrm{S}$, including Maxwell's equations plus the acceleration equation and the consequent inhomogeneous equation (I) that characterize the behaviour of a ferromagnet.

The structure of the London model could then be represented thus:

$$
\mathrm{LM}=\langle\mathrm{S} ;\{\mathbf{E}(\mathrm{x}, \mathrm{t}), \mathbf{B}(\mathrm{x}, \mathrm{t})\}\rangle \text { such that } \mathrm{P}^{\prime}
$$

\footnotetext{
${ }^{16}$ We represent vectorial quantities in bold throughout.
} 
where the principles P' include Maxwell's equations plus the relevant homogenous equation $(\mathrm{H})$ which holds for diamagnets, excluding (I) and the accelerating equation.

Two problems arise with FLBD's theses with this very natural (though highly abbreviated) attempt to represent the two theories in accord with the semantic view. First, these representations capture almost none of the interesting features of how the transition occurred, which both we and they were at pains to stress. French and Ladyman are in agreement with us about the historical details of the transition from the acceleration equation to the London theory, what kinds of motivations the Londons had, what techniques they were familiar with, what information they had to hand, and so forth. But we see no way to express these common assumptions as relations between set-theoretical structures.

The second is a straightforward formal problem. FLBD argue that the significant relations between models can be captured by either isomorphism or by partial isomorphism. Here partial isomorphism does not come into question because the theories do not contain any partial relations. It is also clear that the two structures are not isomorphic since there are superconductors in AET that have $\mathbf{B}(\mathrm{x}, \mathrm{t}) \neq 0$ for $\mathrm{t}$ before the transition to the superconducting state and still have $\mathbf{B}\left(\mathrm{x}, \mathrm{t}^{\prime}\right) \neq 0$ for $\mathrm{t}^{\prime}$ after the transition, whereas in $\mathrm{LM}$ all superconductors have $\mathbf{B}\left(\mathrm{x}, \mathrm{t}^{\prime}\right)=0$ after the transition. There is, of course, a subset of elements of S for which $\mathbf{E}$ and $\mathbf{B}$ obey both P and P', namely the superconductors with $\mathbf{B}(\mathrm{x}, \mathrm{t})=0$ at times previous to the transition to the superconducting state. If we restrict our domain to just these superconductors, we could map out an isomorphism between the two theories. But then we are not really looking at the relations between the London theory and the previous one at all, but merely at a set of cases on which they agree. Nor does this fact seem in any way relevant to the elaborate motivations of the Londons that we agree upon.

We admit that the representation that we have provided so far is not really responsive to FLBD's central idea, however, for they maintain that it is partial relations, and correlatively partial isomorphisms, that provide the powerful tool for capturing relations between models. Where might these partial relations enter? The representation above makes no explicit reference to the characteristic that all highlight 
as central - the difference between modelling superconductors as ferromagnets versus diamagnets. Perhaps we find a way to utilize the FLBD methods if we include these explicitly. Let us try the following then:

$\mathrm{AET}^{\prime}=\left\langle\mathrm{S} ;\left\{\mathbf{E}(\mathrm{x}, \mathrm{t}) ; \mathbf{B}(\mathrm{x}, \mathrm{t}) ; \mathrm{F}_{1}, \mathrm{~F}_{2}, \mathrm{~F}_{3} ; \mathrm{D}_{1}, \mathrm{D}_{2}, \mathrm{D}_{3}\right\}\right\rangle$ such that $\mathrm{P}$

$\mathrm{LM}^{\prime}=\left\langle\mathrm{S} ;\left\{\mathbf{E}(\mathrm{x}, \mathrm{t}) ; \mathbf{B}(\mathrm{x}, \mathrm{t}) ; \mathrm{F}^{\prime}{ }_{1}, \mathrm{~F}_{2}, \mathrm{~F}^{\prime}{ }_{3} ; \mathrm{D}^{\prime}{ }_{1}, \mathrm{D}^{\prime}{ }_{2}, \mathrm{D}^{\prime}{ }_{3}\right\}\right\rangle$ such that $\mathrm{P}^{\prime}$

where $\mathrm{F}$ is the partial property behaves like a ferromagnet (so $F_{l}$ is the set of superconductors that are like ferromagnets; $F_{2}$, the set of those that are not; $F_{3}$, the set about which the model is non-committal); $\mathrm{D}$ the partial property behaves like a diamagnet (so $D_{1}, D_{2}, D_{3}$ are, respectively, the set of superconductors that are, are not and are not declared to be like diamagnets). In AET' all members of S behave like ferromagnets and none like diamagnets, and just the reverse is true for LM'.

But this is just as problematic, for we still have neither an isomorphism nor a partial isomorphism between the two theories. The acceleration equation theory asserted (and this is precisely why it had to be abandoned) that all superconductors conserve the flux during the phase transition; so according to that theory all superconductors are like ferromagnets in obeying I. Thus $\mathrm{F}_{1}$ is the full set $\mathrm{S}$ and so too is $\mathrm{D}_{2}$. In LM' by contrast, $\mathrm{F}_{2}{ }_{2}=\mathrm{S}$ and so is $\mathrm{D}^{\prime}{ }_{1}$. Hence there is no partial isomorphism between the two theories.

Moreover, despite our efforts to introduce partial relations, it seems strained to say we have succeeded. Adopting Hesse's terminology in the way FLBD suggest doing (French and Ladyman, 1999, p. 107), AET' leaves nothing in the neutral analogy of the model, nor does LM'. Hence F, D are not really partial relations after all since in AET' $\mathrm{F}_{2}=\mathrm{F}_{3}=\varnothing$, the empty set and $\mathrm{D}_{1}=\mathrm{D}_{3}=\varnothing$; and similarly, mutatis mutandis, for LM'.

It looks thus as if partial relations are of little help when modelling the practice that took the Londons from the acceleration-equation model to their own. One might hope though that partial relations would apply at least to the relation between Maxwell's 
theory and the Londons' model directly, without the detour via the acceleration equation. For after all Maxwell's equations on their own do not declare themselves regarding superconductivity. Maxwell's theory and the London model are consistent on both our reading and French and Ladyman's. If we were to construe the equations as a model of superconductivity ${ }^{17}$ we would obtain something like:

$$
\mathrm{EM}^{\prime}=\left\langle\mathrm{S} ;\left\{\mathbf{E}(\mathrm{x}, \mathrm{t}) ; \mathbf{B}(\mathrm{x}, \mathrm{t}) ; \mathrm{F},{ }_{1}, \mathrm{~F}{ }^{\prime}{ }_{2}, \mathrm{~F}{ }^{\prime}{ }_{3} ; \mathrm{D}{ }_{1}, \mathrm{D},{ }_{2}, \mathrm{D}{ }^{\prime}{ }_{3}\right\}\right\rangle \text { such that P”, }
$$

where P" = Maxwell's equations. Now, since Maxwell's equations on their own do not state that superconductors are either like ferromagnets or diamagnets, nor do they imply Ohm's law, or the acceleration equation, nor the Londons' equation, it is possible to construe all these as part of the 'neutral' analogy in EM. That is, we could think of F' and D' as partial relations, where all the elements in the domain belong neither to their extension nor outside their extension as follows $\mathrm{F},{ }_{1}=\mathrm{F}{ }_{2}=\mathrm{D}{ }_{1}=$ $\mathrm{D}{ }_{2}=\varnothing, \mathrm{F}_{3}{ }_{3}=\mathrm{D}{ }_{3}=\mathrm{S}$. The problem is that, as we already saw, the London model (LM') has nothing in the neutral or negative part of F' and nothing in the positive or neutral part of D'. So there is no scope for mapping LM' onto EM, while preserving the extensions of F', D', as required by the definition of a partial isomorphism. So partial isomorphism fails to capture even the relation between abstract theory and the more concrete models of the phenomena in this case.

We conclude then that where piecemeal borrowing plays a central role, the advantages of partial over full isomorphism are slim. A partial isomorphism requires that for all $R_{i}$ in the set of relations defining the model, $R_{1}(x y)$ iff $R_{1}$ ' $(f(x) f(y))$ and $\mathrm{R}_{2}(\mathrm{xy})$ iff $\mathrm{R}_{2}$ '( $\left.\mathrm{f}(\mathrm{x}) \mathrm{f}(\mathrm{y})\right)$, so that that every definite assertion of the first model must hold in the second as well (i.e. every relation that is definitely asserted by the first model to hold of a set of objects must still hold of those objects ${ }^{18}$ in the second model and every relation that is definitely asserted not to hold of a set of objects in the first must similarly definitely not hold in the second). So there is no way to leave behind parts of the first model in moving to the second; whatever the first model definitely asserts - either that the relation definitely does hold or that it definitely does not -

\footnotetext{
${ }^{17}$ Obviously we do not think this is a plausible construal - nor is it exceptionally not so: theoretical principles typically do no model anything, but are often useful tools to build models. For a discussion of Newtonian laws along these lines see Giere (1999), chapters 6,7.

${ }^{18}$ Or, more correctly, of their correlates under the mapping function - see Suárez (2005, p. 56).
} 
must still hold in the second model. Given this, it seems impossible to represent piecemeal borrowing with partial relations.

In a recent joint paper Bueno, French and Ladyman (2002) offer a development to their proposal. ${ }^{19}$ They urge that the relation between mathematical theories and the world is often in terms of "partial homo-morphisms". While the application of homomorphism in this context is not new, ${ }^{20}$ we welcome the move from partial isomorphism to partial homomorphism, since, if anything, it strengthens further the instrumentalism of "The Toolbox". However, partial homomorphism too fails to provide a formal scheme to capture piecemeal borrowing.

A partial homomorphism between two partial structures $A=\left\langle D ;\left\{\ldots ; R_{i 1}, R_{i 2}, R_{i 3}\right.\right.$, $; \ldots\}\rangle$ and $\mathrm{B}=\left\langle\mathrm{E} ;\left\{\ldots ; \mathrm{R}^{\prime}{ }_{11}, \mathrm{R}^{\prime}{ }_{\mathrm{i} 2}, \mathrm{R}^{\prime}{ }_{\mathrm{i} 3} ; \ldots\right\}\right\rangle$ is a function $\mathrm{f}: \mathrm{D} \rightarrow \mathrm{E}$, such that for every $\mathrm{x}$ and $\mathrm{y} \in \mathrm{D}, \mathrm{R}_{\mathrm{i} 1}(\mathrm{x}, \mathrm{y})$ only if $\mathrm{R}^{\prime}{ }_{\mathrm{i} 1}(\mathrm{f}(\mathrm{x}), \mathrm{f}(\mathrm{y}))$ and $\mathrm{R}_{\mathrm{i} 2}(\mathrm{x}, \mathrm{y})$ only if $\mathrm{R}^{\prime}{ }_{\mathrm{i} 2}(\mathrm{f}(\mathrm{x}), \mathrm{f}(\mathrm{y}))$. Note that objects in the second model can now possess relations they do not have no in the first, since it is not required in a partial homomorphism that $\mathrm{R}^{\prime}{ }_{11}(\mathrm{f}(\mathrm{x}), \mathrm{f}(\mathrm{y})) \rightarrow \mathrm{R}_{\mathrm{i} 1}(\mathrm{x}$, y). But this is still not sufficient to capture the fact that there may be relations positively ascribed to objects in the old model - like behaving like a ferromagnet and satisfying equation (I) - that we wish to deny in the new.

\section{Structures for the toolbox.}

In many of the writings by FLBD our work is used as a foil against which to present their own developments of the semantic view. They attempt to show that the kinds of worries we have can be accommodated once looser relations among models like the ones they introduce are allowed. This suggests that they take "The Toolbox" to oppose the semantic conception. Is this correct?

That depends on the order of the quantifiers. The semantic conception is often taken by many, including FLBD, to say that there are a set of relations $R$ definable between

\footnotetext{
${ }^{19}$ They claim to be responding to Suárez (1999), although Suárez's critique of isomorphism is his (1997).

${ }^{20}$ Its introduction goes back, at least, to Suppes' early writings on the topic. See Suppes (1962b); and Mundy (1986); and it is ubiquitous in the writings of the German structuralists (see note 40 for references).
} 
models (like isomorphism, partial isomorphism, and so forth) such that for any good theory $\mathrm{T}$ there is a set of models $T$ that constitutes $\mathrm{T}$ and such that for any representative model $M^{21}$ constructed 'from' T, $M$ is related to $T$ via $R$. It is then a question of figuring out what kind of morphism $R$ is in general, and it is here that different variants of the semantic conception differ.

We are suspicious of such a strong claim in general. But we do not quarrel with the assumption that formalisms may be useful in specific cases to highlight specific points of contact between theories and models. So we see no reason to oppose a different claim with the quantifiers differently ordered: for any theory $\mathrm{T}$ and any representative model $M$ constructed using it, there is a set of models $T(M)$ and there are a set of relations $R(T, M)$ such that $M$ is related to $T$ via $R(T, M)$. This weak claim is plausible for different particular cases, but cannot be used to defend the stronger claims by FLBD to have provided a universal account of modelling.

Applying the weak claim to the superconductivity example would mean rejecting that the same type of morphism (whether partial isomorphism, homomorphism, or any other variety) must hold between the structures exemplified by the theory and the two models at issue. That is, even if we succeeded at formulating structures for classical electromagnetism, the London model and the acceleration equation model, we would refrain from adopting a particular relation $R$ which must hold between all of them.

We might then adopt a much more liberal account of the relation between theories and models as a loose hierarchy of structures, along the lines of Suppes' original ideas (Suppes, 1962a, 2002). ${ }^{22}$ The main claim, however, is weaker than any partial isomorphism - implied by French and Ladyman (1997), or partial homomorphism explicitly claimed by Bueno, French and Ladyman (2002). On this account we simply

\footnotetext{
${ }^{21}$ To be more accurate, we should allow that the representative models in question do not themselves already come represented as models - set-theoretic structures - in the sense of the semantic view. So we need an extra quantification to say that for every representative model $\mathrm{M}$ there is a (set-theoretic) model $M$ that represents it. We do not know whether FLBD would want to assume that for every representative model there is only one formal model to represent it, as they seem to suppose for the models that constitute theory, or whether with us they will allow very different formalizations for different purposes. At any rate we suppress this issue in the text in order to concentrate on the issue of formalizing theory.

${ }^{22}$ Kaiser (1991) follows Suppes in providing a similar schema for the relation between data models and phenomena. The remainder of this section borrows heavily from Suárez (1997 and 2005).
} 
refrain from making any assumptions at all regarding the structural relations between theory and the different models.

In this framework a theoretical model $T_{k}$ sits at the top of a hierarchy of models of phenomena $\mathrm{M}_{1}$ to $\mathrm{M}_{\mathrm{k}-1}$ :

$$
\begin{aligned}
& \mathrm{T}_{\mathrm{k}}=\left\langle\mathrm{D}_{\mathrm{k}} ;\left\{\mathrm{R}_{\mathrm{k}}^{1}, \ldots, \mathrm{R}_{\mathrm{k}}^{\mathrm{m}}\right\}\right\rangle \\
& \mathrm{M}_{\mathrm{k}-1}=\left\langle\mathrm{D}_{\mathrm{k}-1} ;\left\{\mathrm{R}_{\mathrm{k}-1}^{1}, \ldots, \mathrm{R}_{\mathrm{k}-1}^{\mathrm{n}}\right\}\right\rangle \\
& \ldots . \\
& \ldots . \\
& \mathrm{M}_{1}=\left\langle\mathrm{D}_{1} ;\left\{\mathrm{R}_{1}^{1}, \ldots, \mathrm{R}^{\mathrm{p}}{ }_{1}\right\}\right\rangle \\
& \mathrm{S}_{0}=\left\langle\mathrm{D}_{0} ;\left\{\mathrm{R}_{0}{ }_{0}, \ldots, \mathrm{R}^{\mathrm{q}} 0\right\}\right\rangle
\end{aligned}
$$

We might suppose the bottom layer's structure to have as its domain $\mathrm{D}_{0}$ only objects that can be directly measured. The next structure up is defined by introducing a set of new relations over an expanded or completely different domain (there is therefore no requirement that $\mathrm{D}_{\mathrm{i}} \subseteq \mathrm{D}_{\mathrm{i}+1}$ ). The whole hierarchy is defined in a number of steps, or layers, each of which may involve a change of elements in the domain, or a change in the set of relations. These operations are intended to represent scientific practice; hence they correspond to operations and redefinitions of the domain that are performed in the course of scientific research. The passage from one structure to the next one up is not grounded upon any features of the structures themselves, nor upon their relations but upon so-called 'inference tickets' (Kaiser, 1991) which come in two kinds: as warrant for a re-description of the domain, or as legitimation of new types of operations and computations. Theoretical knowledge is involved - although not necessarily knowledge derived from one and the same theory. 'Inference tickets' are provided by probing techniques and instruments, error theory, test-methods to eliminate useless samples, statistical techniques for the treatment of data, etc.

On this kind of hierarchy of structures the construction of models of phenomena is independent of theory in just the way we describe. It is a very unrestrictive structuralism since it forgoes the requirement that the structures should exhibit 
correspondences of any particular type. The higher structure need not embed, fully or partially, the lower structures of the hierarchy. Nor is there any expectation that there will be full or partial isomorphisms or homomorphisms between them.

Let us then characterise more precisely from this structural point of view the relations between Maxwell's theory, the acceleration equation theory, and the London model:

$\mathrm{EM}=\langle\mathrm{S} ;\{\mathbf{E}(\mathrm{x}, \mathrm{t}) ; \mathbf{B}(\mathrm{x}, \mathrm{t})\}\rangle$ such that P”,

$\mathrm{AET}=\langle\mathrm{S} ;\{\mathbf{E}(\mathrm{x}, \mathrm{t}), \mathbf{B}(\mathrm{x}, \mathrm{t})\}\rangle$ such that $\mathrm{P}$

$\mathrm{LM}=\langle\mathrm{S} ;\{\mathbf{E}(\mathrm{x}, \mathrm{t}), \mathbf{B}(\mathrm{x}, \mathrm{t})\}\rangle$ such that $\mathrm{P}^{\prime}$

On this account he inference ticket that supports the move from EM to AET is the assumption that superconductors behave like ferromagnets, which justifies including Ohm's law among the principles for AET; that for LM is the alternative assumption that superconductors behave like diamagnets. These are supported by the observations of resistanceless flow and, for the second case, the expulsion of the magnetic field, in conjunction with all the past experience in treating electromagnetic phenomena that indicate that these are good analogies given the two phenomena. In this case the domains for the models are all the same but there is no requirement that the domains, or relations, have the same cardinality, or that they are nested in any way. Although we do not deploy them in this case, the models may well contain partial as well as full relations. But there is no requirement that there be a mapping from the domain of EM, or AET to that of LM that preserves partial or full relations, etc. There is in other words no reason to expect an isomorphism, partial isomorphism, partial homomorphism or any other morphism between the Londons' model (LM), and Maxwell's theory (EM), or the acceleration equation theory (AET). This allows for properties in the model that are denied in the phenomena and vice versa; it also allows theories to stay silent about certain features of the phenomena.

But notice then that this framework can account for the piecemeal borrowing characteristic of the practice of modelling precisely because it leaves out of the 
description everything that is of interest for our thesis in "The Toolbox", namely the actual reasons scientists advance for building a new model. The framework leaves out precisely the Londons' reasoning in deriving the London model out of the acceleration equation theory - which is what we have been claiming is crucial to assess the theory-driven view. Hence we see that an appropriate structural characterisation of this practice can at best describe correctly the products of the modelling practice (i.e. the models themselves); while necessarily leaving out the intellectual processes that lead to those models.

\section{Conclusions}

We have not found any reasons in the work of FLBD to give up any of our claims in "The Toolbox". In particular we stand firmly by the claim that successful model building is generally theory-independent in just the way we describe. We also stand by the claim that the London superconductivity example is a good illustration of this kind of theory independence. With respect to FLBD's positive claims about partial morphisms holding between theories and models, we have pointed out that contrary to their repeated claims FLBD have not actually provided a reconstruction of the London model example in their framework of partial structures. Moreover in our best attempts to sketch out how to do so on their behalf, partial structures as defined by FLBD will not do the job demanded. In particular they do not seem after all well suited to reconstructing the piecemeal borrowing deployed by the Londons and so central to much successful modelling practice. This invites serious scepticism that any one type of formal set-theoretic relations can be employed to understand the vast range of relationships among related models that matter to successful model-building.

Note that we have been going along with FLBD in presupposing, for the sake of argument, that the semantic view provides the right account not just of the abstract nature of theory, but even the practice of theorising. The latter at least may now be doubted, and we do doubt it. The point at issue is whether good theories have a canonical formal representation that gets at the core of what they say and that is the 
same for all, or at least most, practical purposes of theorising. ${ }^{23}$ We are suspicious of this because we think that in practice theorising is constituted by a heterogeneous mix of journal articles, textbooks, lectures, $\mathrm{PhD}$ seminars, practices, techniques, explicit explanations, implicit, half-formed understandings and the like. This is a lesson that the last two decades of science studies work brings forcefully home (Cf. Galison, 1997). What we know 'theoretically' is recorded in a vast number of places in a vast number of different ways - not just in words and formulae but in machines, techniques, experiments and applications as well. Attempts to winnow out of this a single canonical formulation are likely either to miss most of what constitutes the use and application of theory in practice, or will be so abstract as to be almost empty of content.

\section{$\underline{\text { References: }}$}

Balzer, W., Moulines, C.U. \& Sneed, J. (1987). An architechtonic for science. Dordrecht: Reidel.

Balzer, W. \& Moulines, C.U. (1996). Structuralist theory of science: Focal issues, new results. Berlin: Walter de Gruyter.

Bueno, O. (1997). Empirical adequacy: A partial structures account. Studies in the History and Philosophy of Science, 28, pp. 585-610.

Bueno, O., S. French and J. Ladyman, (2002). On representing the relationship between the mathematical and the empirical. Philosophy of Science, 69, pp. 497-518.

Cartwright, N. (1983). How the laws of physics lie. Oxford: Oxford University Press.

\footnotetext{
${ }^{23}$ Of course most people we suppose will not assume that there is a single right formulation down to the last detail. But it does seem to be supposed by semantic theorists that there will be a close similarity among different acceptable formulations.
} 
Cartwright, N., T. Shomar and M. Suárez (1995). The tool box of science. In Herfel, W., W. Krajewski, I. Niiniluoto and R. Wojcicki (eds.), Theories and models in scientific processes (pp. 137-149). Amsterdam: Rodopi.

Da Costa, N. and S. French (2000). Models, theories and structures: Thirty years on. Philosophy of Science Supplement, 67, pp. 116-127.

Da Costa, N. and S. French (2003). Science and partial truth. New York: Oxford University Press.

Dahl, P. F. (1993). Superconductivity: Its historical roots and development from mercury to the ceramic oxides. New York: American Institute of Physics.

French, S. (1999). The phenomenological approach to physics. Studies in the History and Philosophy of Modern Physics, 30, pp. 267-281.

French, S. and Ladyman, J. (1997). Superconductivity and structures: Revisiting the London account. Studies in the History and Philosophy of Modern Physics, 28, pp. 363-393.

French, S. and J. Ladyman (1998). A semantic perspective on idealization in quantum mechanics. In N. Shanks (ed.), Idealization VIII: Idealization in contemporary physics. Poznán Studies in the Philosophy of the Sciences and the Humanities. Amsterdam: Rodopi.

French, S. and Ladyman, J. (1999). Reinflating the semantic approach. International Studies in the Philosophy of Science, 13, pp. 103-121.

Friedman, M. (1953). Essays in positive economics. Chicago: Chicago University Press.

Frisch, M. (2000). (Dis-)solving the puzzle of the arrow of radiation. British Journal for the Philosophy of Science, 51, pp. 381-410. 
Frisch, M. (2005). Inconsistency, asymmetry and non-locality: A philosophical exploration of classical electrodynamics. Oxford: Oxford University Press.

Galison, P. (1999). Image and logic. Chicago: University of Chicago Press.

Gavroglu, K. (1995). Fritz London: A scientific biography. Cambridge: Cambridge University Press.

Giere, R. (1988). Explaining science: A cognitive approach. Chicago: University of Chicago Press.

Kaiser, M. (1991). From rocks to graphs: The shaping of phenomena. Synthese, 89, pp. 111-133.

Ladyman, J. (1998). What is structural realism? Studies in the History and Philosophy of Science, 29, pp. 109-24.

Ladyman, J. (2002). Understanding Philosophy of Science. London and New York: Routledge.

McMullin, E. (1985). Galilean idealization. Studies in the History and Philosophy of Science, 16, pp. 247-263.

Morgan, M. S. (1999). Learning from models. In M. Morgan and M. Morrison (eds.), Models as mediators (pp. 347-388). Cambridge: Cambridge University Press.

Morgan, M. S. and M. Morrison (eds.), (1999). Models as mediators: Perspectives on the natural and social sciences. Cambridge: Cambridge University Press.

Morrison, M. (1999). Models as autonomous agents. In M. Morgan and M. Morrison (eds.), Models as mediators (pp. 38-65). Cambridge: Cambridge University Press.

Morgan, M. (forthcoming), Models as Modelling, manuscript. 
Mundy, B. (1986). On the general theory of meaningful representation. Synthese, 67, pp. 391-437.

Nowak, L. (1980). The structure of idealization: Towards a systematic interpretation of the Marxian idea of science. Dordrecht: Reidel.

Post, H. (1971). Correspondence, invariance and heuristics. Studies in the History and Philosophy of Science, 2, pp. 213-255.

Suárez, M. (1995). How theories save phenomena: A case against embedding. Talk given at the British Society for the Philosophy of Science annual meeting, University of Leeds, September 2005.

Suárez, M. (1997). Models of the world, data-models and the practice of science, $\mathrm{PhD}$ Thesis, London School of Economics, August 1997.

Suárez, M. (1999). The role of models in the application of scientific theories: Epistemological implications. In M. Morgan and M. Morrison (eds.), Models as mediators (pp. 168-196). Cambridge: Cambridge University Press.

Suárez, M. (2005). The semantic view, empirical adequacy, and application. Crítica, 37, pp. 29-63.

Suppes, P. (1962a). Models of data. In Nagel et al (eds.), Logic, Methodology and Philosophy of Science: Proceedings of the 1960 International Congress. Stanford: Stanford University Press, pp. 252-261.

Suppes, P. (1962b). Set-theoretical structures in science, Stanford University preprint, reprinted as Suppes (2002).

Suppes, P. (2002). Representation and invariance of scientific structures. Stanford: CSLI publications.

Van Fraassen, B. (1989). Laws and symmetry. Oxford: Oxford University Press. 
